\title{
Social Transmission and Shared Reality in Cultural Dynamics
}

Yoshihisa Kashima, Melbourne School of Psychological Sciences, The University of Melbourne Boyka Bratanova, School of Management, University of St Andrews

Kim Peters, School of Psychology, University of Queensland

Main Text Word Count: 2272

\section{Abstract (112 words)}

Micro cultural dynamics are concerned with the mechanisms of transmission, retention, and modification of cultural information in social networks. When interacting individuals mutually recognize that they share psychological reactions to given cultural information, it may be grounded as an aspect of their shared reality under specifiable conditions. The interpretation of cultural information as socially verified shared reality provides a basis for further dissemination of the information and coordinated social action. We review the recent literature that supports this general contention, while highlighting the role of emotion - a somewhat under-recognized aspect of shared reality research - and emphasizing the mediating role of cultural dynamics in the mutual constitution of social reality and shared reality.

\section{Acknowledgment}

Preparation for this manuscript was facilitated by grants from the Australian Research Council (DP160102226 and DP160102231) to YK. 


\section{Introduction}

Culture is a set of available information that is transmitted non-genetically in a human population. The social transmission of cultural information between individuals in situ is central to cultural dynamics - the formation, maintenance, and transformation of culture over time. Interpersonal communication, as a type of cultural transmission, is critical for the establishment of shared reality. In this paper, we will spell out the ways in which shared reality is implicated in cultural transmission and therefore in cultural dynamics.

Cultural transmission is composed of four subprocesses: production, grounding, interpretation, and memory. Individuals produce a communicable representation of cultural information, ground it to the common ground (i.e., add it to the information actually, and perceived to be, shared with their interaction partners), interpret the grounded information (i.e., transform it into a mental representation), and commit it to memory for future use [1]. Of these, grounding establishes mutuality. It occurs when the sender and receiver both recognize that their understanding of the communicated information is sufficiently similar to carry out their joint activity in the context $[2,3]$.

We argue that shared reality affects cultural transmission by changing the way in which grounded information is interpreted. Specifically, in the presence of shared reality, this information is tagged as socially verified "fact" (Figure 1). Importantly, socially verified information is more likely to spread through social networks (Figure 2). In this way, shared reality links cultural transmission to macro-level cultural diffusion. In this article, we will first review relevant literature to flesh out these propositions and supportive evidence, and then examine the role of emotion in this process.

\section{Grounding and shared reality in dyadic context}

When cultural information is grounded in a dyadic context, the sender and receiver accept the grounded information for the purpose of their interaction in the current context. At this point, the information is not necessarily taken to be a verified "fact" that is generally applicable to everyone under all circumstances. However, if the sender and receiver achieve a shared psychological response to the information and mutually recognize this as fulfilling either epistemic motives (i.e., to find out what is true) or relational ones (i.e., to form or maintain meaningful relationships), then the grounded information becomes a shared reality [4; also see Echterhoff \& Higgins, this issue]. It is then interpreted as a "fact" that can be generalized beyond the immediate context (Figure 1). Thus, shared reality processes tag the cultural information as a socially verified fact.

In a dyadic context, the sender's motive to achieve mutuality with the receiver can result in communicating biased information [i.e., audience tuning; 5, 6]; the sender's interpretation that the communicated information has been socially verified with the receiver, and thus established shared reality biases the sender's memory about the communicated social object [7].

In addition, the sender's audience tuned message can bias its receiver's cognitions as well. Stukas, Bratanova, and their colleagues [8] have shown that, if a sender communicates biased information about a target group, this can influence the receiver's attitudes and behavioral intentions towards the target when the receiver reported that a sense of shared reality was established. This research highlights the role of shared reality as a necessary condition to produce the actual sharing of psychological responses and to create biased collective cognitions, which neither the sender nor the receiver held before [9].

Furthermore, grounding and establishing shared reality about a social category (i.e., a group of people) can essentialize the social category. Essentialism is a belief that a category has an underlying immutable essence, which causes its exemplars to possess their surface-level characteristics $[10,11]$. Within the context of a social category, it amounts to beliefs that members of the social category typically have the same underlying essence (e.g., hostile), which causes them to behave in certain ways (e.g., hitting and injuring people). Kashima and colleagues [12] found that grounding the information about a novel group can strengthen the communicators' beliefs that this 
group's members share the same immutable characteristics. Wagner, Holtz, and Kashima [13] suggested that this may be due to the communicators' use of "essence-tools", linguistic expressions that convey the existence of an immutable essence, including nominalized category labels [i.e., noun phrases like "carrot eaters" rather than "people who eat lots of carrots"; 14] and generics [i.e., expressions like "Zarpies are scared of ladybugs" which describe general characteristics of a category; 15].

\section{Cultural transmission beyond dyad}

Once cultural information is grounded and interpreted as the dyad's shared reality, the cultural information is regarded as a fact that applies to everyone under all circumstances. One of the consequences is that the cultural information can then be further disseminated, diffusing through social networks in the broader community and population (Figure 2). Different experimental paradigms can simulate the diffusion of cultural information through social networks with different structural properties.

When stimuli are communicated repeatedly by a sender to multiple receivers who belong to the same group (repeated reproduction, Figure 3 ), this simulates a "hub" (highly central node) in a social network. If the sender audience-tunes to the first receiver's attitude and sends a biased message, similarly biased messages tend to be repeated in subsequent communications to other receivers, provided that a group level shared reality is perceived to exist [16]. Interestingly, these repeated communications were biased even though the sender's memories about the topic of communication were in fact more accurate and less biased than their message. Communicating a biased message even when more accurate recollections were available to the sender suggests that perceived group-level shared reality facilitates the diffusion of potentially biased cultural information.

When stimuli are communicated from one person to another in a chain (serial reproduction, Figure 2), this simulates a linear communication chain. Recent research has found that initially unstructured information becomes more structured, more meaningful for the communicators, and easier to comprehend and communicate [17] as it is transmitted through the chain. This general mechanism can create social categories and associate them with a set of attributes, giving rise to group boundaries and outgroup stereotypes [18]; it can also amplify negative social evaluations and inferences in the context of conflict, resulting in strongly polarized views and prejudice even in the absence of justifiable evidence [19]. For a recent review of human and animal research on serial reproduction, see [20].

There are two mechanisms that potentially enhance the fidelity of cultural transmission by enhancing the sense of shared reality about the information. The first is allowing the sender and receiver to communicate bidirectionally. Tan and Fay [21] compared the standard serial reproduction chain to a chain with a bidirectional interaction and found that senders re-produced more information more accurately when given the chance to engage in grounding (e.g., seeking and giving clarifications and elaborations). Interestingly, receivers also exhibited superior recall in this treatment, suggesting that mutuality is better achieved when opportunities for active grounding in communication are afforded. The second mechanism involves the receipt of cultural information from multiple senders. When this occurs, receivers tend to exhibit superior memory for the information [22]. While this can be partly attributed to the richer input, it also speaks to the importance of social validation - integral to shared reality - whereby information that is corroborated by multiple sources may be considered more valid and therefore worthy of retention in memory.

\section{Collective Common Ground, Collective Shared Reality, and Cultural Dynamics}

If cultural information spreads beyond dyads (mass media and other communication channels can play a significant role here) and its members have a mutual sense of shared reality about it within the population (collective shared reality), this information enters into the collective 
common ground (i.e., information that people in a population take for granted as shared within the population). Information that is congruent with the common ground tends to be preferentially selected for further communication even if equally good or even better quality alternatives are available [17, 23-27]. For instance, Fast and colleagues [23] demonstrated that when senders were tasked to initiate a conversation about baseball, they showed a strong preference towards choosing well-known but underperforming players as a topic of their conversation over lesser known players whose recent performance was outstanding. Because familiarity facilitates grounding and the achievement of mutuality [17], it can therefore facilitate cultural diffusion.

Chiu and colleagues [28] used the term, intersubjective culture, to refer to culture that is collectively seen to be shared in a population. Several studies showed that intersubjective culture influences values $[29,30]$ as well as other psychological tendencies like thinking styles, attribution, and compliance [31]. Shared reality can also play a role in maintaining the values and beliefs perceived to be shared within a collective. Receiving information that denounced beliefs perceived to be widely shared within a collective elicited disbelief of the information, and intensified positive and affirmative evaluation of the existing cultural beliefs, thus preserving collective shared reality [32].

\section{Emotion in Cultural Transmission and Shared Reality}

There is growing evidence that emotion plays a significant role in cultural dynamics. In particular, emotional representations - those relating to potential threats or opportunities in the environment $[33,34]$ - are especially likely to characterise the collective common ground. This is because when people experience an emotional response to an event, they are highly likely to talk about this event, thereby establishing the mutuality of their emotional reaction with others and, thus, a shared reality [35-38].

Whether the event concerns the self or another person, legend or fact, the more it arouses emotion, the more willing people seem to disseminate it through social networks [e.g., 39]. There is increasing evidence for the preferential dissemination of information within large collectives. For instance, the emotionality of the information was found to positively predict the tendency for people to share new articles in the New York Times [39], to pass on messages about German state elections on twitter [40] and to retweet messages about same-sex marriage and climate change [41].

Although the increased communicability of emotional information appears to be a robust finding, the precise emotions that drive communication in any particular context appears to vary. For instance, while some studies have found a communication advantage for negatively valenced information like disgust and fear $[42,43]$, others have failed to find a simple valence effect [35] or have found an advantage for positive information [e.g., 39]. Therefore, it is likely that in line with the idea of the situated nature of cultural dynamics, relevance of cultural information is likely to vary with the specific social context. For instance, although Eriksson, Coultas, and de Barra [44] were able to replicate previous findings around the communicability of disgust in North American samples, there was no evidence for this effect among Indians who showed a greater preference for communicating fear arousing content.

There is a reason to expect that emotional information, once communicated, is especially likely to be incorporated into shared reality. First, people appear to talk about emotional events, at least in part, out of a desire to establish a sense of shared reality with their audience. Specifically, Duprez et al. [45] found that almost half of their sample reported that they had told another person about an emotional event in order to clarify their understanding of, and appropriate response to, the event, or to establish a shared perspective of it. Indirect evidence that communicators look to establish shared reality comes from work that shows that communicators who do not have their emotional response verified by their audience perceive this audience more negatively [e.g., 46]. Second, people's tendencies to express the emotions that they feel (whether verbally or nonverbally) makes it likely that any actual congruence in emotional response will be mutually recognised as such and contribute to a sense of shared reality. In line with this possibility, Peters et 
al. [47] found that the amount of time dyads spent discussing a positive or negative norm violation (a type of event that can arouse emotions) had a positive indirect association with their sense of shared reality.

Emotional events that are incorporated into collective common ground are also likely to continue to disseminate through networks of communicators. In this way, emotional experiences, as well as representations of the event's significance and appropriate behavioural responses, can become shared within collectives [48]. This shared reality means that the significance of ambiguous events is likely to be resolved in ways that amplify perceptions of their significance. In line with this, Yzerbyt et al. [49] found that allowing students to discuss a controversial educational policy increased their perceptions that the policy was unfair and their levels of anger relative to students who were not allowed to discuss it. As well as helping people to coordinate their collective responses to threats and opportunities, it will predispose members of the network to categorise themselves as members of a group that can achieve their emotion-related goals $[48,50]$.

\section{Conclusion}

Shared reality processes provide a critical link in connecting micro-level cultural transmission to macro-level diffusion of cultural information, and therefore formation, maintenance, and transformation of the distribution of cultural information in a population. We suggest that shared reality acts as a cognitive tag, signifying that the transmitted and grounded cultural information has been socially verified as a "fact" and therefore is applicable in the future to others under other circumstances. Once established as shared reality, the cultural information can then be transmitted further beyond the particular context of cultural transmission to others in the population and thus diffuse through social networks. Recent work has pointed to the significance of emotion as a driver of cultural dynamics. Although the role of emotion in shared reality processes is not well understood, we suggest that it may be critical to investigate it in future research. 


\section{Outstanding Interest $(* *)$}

Duprez, Christophe [45]

The authors build on evidence that people share emotional content by explaining why they do so. This paper discusses the development and testing of a 7-factor social sharing motives scale. In addition to intra-individual motives, like venting, several of these factors broadly relate to a desire to establish a shared reality.

Stukas, Bratanova [9]

An examination of the communication processes involved in the creation of cultural information (i.e., social stereotypes) from both sender's and receiver's perspectives. The studies highlight the role of grounding efforts, in the form of audience tuning, and shared reality in the creation of biased cognitions and behavioural tendencies towards a social group that neither communication partner held before.

\section{Special Interest $(*)$}

Fast, Heath [23]

Research of high ecological validity demonstrating how the pursuit of grounding in communication contributes to the maintenance of culturally prominent information.

Hunzaker [27]

Deflection of information is the extent to which the information is felt to be strange and incongruous given the culturally shared affective meaning about the topic of the communication. The paper shows how to measure deflection, and provides evidence that people attempt to reduce deflection in retelling a cultural narrative, thereby contributing to cultural maintenance.

Martin, Hutchison [18]

A serial reproduction study that demonstrates the emergence of meaningful stereotypes through sheer social transmission processes.

Yzerbyt, Kuppens [49]

This paper shows how allowing groups to communicate about a negative event (versus an irrelevant topic) leads to more extreme emotional reactions and appraisals, and well as higher levels of groupbased identity. 


\section{References}

1. Kashima, Y., Cultural dynamics. Current Opinion in Psychology, 2016. 8: p. 93-97.

2. Kashima, Y., Meaning, grounding, and the construction of social reality. Asian Journal of Social Psychology, 2014. 17(2): p. 81-95.

3. Miller, T., et al., Logics of common ground. Journal of Artificial Intelligence Research, 2017. 58: p. 859-904.

4. Echterhoff, G., E.T. Higgins, and J.M. Levine, Shared reality experiencing commonality with others' inner states about the world. Perspectives on Psychological Science, 2009. 4(5): p. 496-521.

5. Higgins, E.T., Achieving 'shared reality' in the communication game: A social action that create meaning. Journal of Language and Social Psychology, 1992. 11(3): p. 107-131.

6. Higgins, E.T., "Saying is believing" effects: When sharing reality about something biases knowledge and evaluations, in Shared cognition in organizations: The management of knowledge, L.L. Thompson, J.M. Levine, and D.M. Messick, Editors. 1999, Erlbaum: Mahwah, NJ. p. 33-49.

7. Echterhoff, G., R. Kopietz, and E.T. Higgins, Shared reality in intergroup communication: Increasing the epistemic authority of an out-group audience. Journal of Experimental Psychology: General, 2017. 146(6): p. 806-825.

8. Stukas, A.A., et al., Confirmatory processes in attitude transmission: The role of shared reality. Social Influence, 2010. 5(2): p. 101-117.

9. Stukas, A.A., et al., Confirmatory processes in attitude transmission: The role of shared reality. Social Influence, 2010. 5(2): p. 101-117.

10. Medin, D.L. and A. Ortony, Psychological essentialism, in Similarity and anlogical reasoning, S. Vosniadou and A. Ortony, Editors. 1989, Cambridge University Press: New York, NY. p. 179-195.

11. Gelman, S.A., Psychological essentialism in children. Trends in Cognitive Sciences, 2004. 8(9): p. 404-9.

12. Kashima, Y., et al., Communication and essentialism: Grounding the shared reality of a social category. Social Cognition, 2010. 28(3): p. 306-328.

13. Wagner, W., P. Holtz, and Y. Kashima, Construction and deconstruction of essence in representating social groups: Identity projects, stereotyping, and racism. Journal for the Theory of Social Behaviour, 2009. 39(3): p. 363-383.

14. Gelman, S.A. and G.D. Heyman, Carrot-eaters and creature-believers: The effects of lexicalization on children's inferences about social categories. Psychological Science, 1999. 10(6): p. 489-493.

15. Rhodes, M., S.-J. Leslie, and C.M. Tworek, Cultural transmission of social essentialism. Proceedings of the National Academy of Sciences of the United States of America, 2012. 109(34): p. 13526-31. 
16. Bratanova, B.A. and Y. Kashima, The "saying is repeating" effect: Dyadic communication can generate cultural stereotypes. Journal of Social Psychology, 2014. 154(2): p. 155-174.

17. Clark, A.E. and Y. Kashima, Stereotypes help people connect with others in the community: A situated functional analysis of the stereotype consistency bias in communication. Journal of Personality and Social Psychology, 2007. 93(6): p. 1028-1039.

18. Martin, D., et al., The spontaneous formation of stereotypes via cumulative cultural evolution. Psychological Science, 2014. 25(9): p. 1777-1786.

19. Lee, T.L., M.J. Gelfand, and Y. Kashima, The serial reproduction of conflict: Third parties escalate conflict through communication biases. Journal of Experimental Social Psychology, 2014. 54(1): p. 68-72.

20. Whiten, A., C.A. Caldwell, and A. Mesoudi, Cultural diffusion in humans and other animals. Current Opinion in Psychology, 2016. 8: p. 15-21.

21. Tan, R. and N. Fay, Cultural transmission in the laboratory: agent interaction improves the intergenerational transfer of information. Evolution and Human Behavior, 2011. 32(6): p. 399-406.

22. Eriksson, K. and J.C. Coultas, The advantage of multiple cultural parents in the cultural transmission of stories. Evolution and Human Behavior, 2012. 33(4): p. 251-259.

23. Fast, N.J., C. Heath, and G. Wu, Common ground and cultural prominence: How conversation reinforces culture. Psychological Science, 2009. 20(7): p. 904-911.

24. Lyons, A. and Y. Kashima, How are stereotypes maintained through communication? The influence of stereotype sharedness. Journal of Personality and Social Psychology, 2003. 85(6): p. 989-1005.

25. Kashima, Y., Maintaining cultural stereotypes in the serial reproduction of narratives. Personality and Social Psychology Bulletin, 2000. 26(5): p. 594-604.

26. Hunzaker, M.B.F., Making sense of misfortune: Cultural schemas, victim redefinition, and the perpetuation of stereotypes. Social Psychology Quarterly, 2014. 77(2): p. 166-184.

27. Hunzaker, M.B.F., Cultural Sentiments and Schema-Consistency Bias in Information Transmission. American Sociological Review, 2016. 81(6): p. 1223-1250.

28. Chiu, C.-Y., et al., Intersubjective culture: The role of intersubjective perceptions in crosscultural research. Perspectives on Psychological Science, 2010. 5(4): p. 482-493.

29. Fischer, R., Congruence and functions of personal and cultural values: Do my values reflect my culture's values? Personality and Social Psychology Bulletin, 2006. 32(11): p. 1419-31.

30. Wan, C., et al., Perceived cultural importance and actual self-importance of values in cultural identification. Journal of Personality and Social Psychology, 2007. 92(2): p. 337-54.

31. Zou, X., et al., Culture as common sense: Perceived consensus versus personal beliefs as mechanisms of cultural influence. Journal of Personality and Social Psychology, 2009. 97(4): p. 579-97. 
32. Wan, C., C.J. Torelli, and C.-Y. Chiu, Intersubjective consensus and the maintenance of normative shared reality. Social Cognition, 2010. 28(3): p. 422-446.

33. Frijda, N.H., P. Kuipers, and E. ter Schure, Relations among emotion, appraisal, and emotional action readiness. Journal of Personality and Social Psychology, 1989. 57(2): p. 212228.

34. Smith, C.A. and R.S. Lazarus, Appraisal components, core relational themes, and the emotions. Cognition and Emotion, 1993. 7(3-4): p. 233-269.

35. Stubbersfield, J.M., J.J. Tehrani, and E.G. Flynn, Chicken tumours and a fishy revenge: Evidence for emotional content bias in the cumulative recall of urban legends. Journal of Cognition and Culture, 2017. 17(1-2): p. 12-26.

36. Heath, C., C. Bell, and E. Sternberg, Emotional selection in memes: The case of urban legends. Journal of Personality and Social Psychology, 2001. 81(6): p. 1028-1041.

37. Peters, K., Y. Kashima, and A.E. Clark, Talking about others: Emotionality and the dissemination of social information. European Journal of Social Psychology, 2009. 39(2): p. 207-222.

38. Rimé, B., Emotion elicits the social sharing of emotion: Theory and empirical review. Emotion Review, 2009. 1(1): p. 60-85.

39. Berger, J. and K.L. Milkman, What makes online content viral? Journal of Marketing Research, 2012. 49(2): p. 192-205.

40. Stieglitz, S. and L. Dang-Xuan, Emotions and information diffusion in social media-sentiment of microblogs and sharing behavior. Journal of Management Information Systems, 2013. 29(4): p. 217-248.

41. Brady, W.J., et al., Emotion shapes the diffusion of moralized content in social networks. Proceedings of the National Academy of Sciences of the United States of America, 2017. 114(28): p. 7313-7318.

42. Bebbington, K., et al., The sky is falling: Evidence of a negativity bias in the social transmission of information. Evolution and Human Behavior, 2017. 38(1): p. 92-101.

43. Brennan, E., et al., Why do smokers talk about antismoking campaigns? Predictors of the occurrence and content of campaign-generated conversations. Health Communication, 2016: p. Advance online publication.

44. Eriksson, K., J.C. Coultas, and M. de Barra, Cross-cultural differences in emotional selection on transmission of information. Journal of Cognition and Culture, 2016. 16(1-2): p. 122-143.

45. Duprez, C., et al., Motives for the social sharing of an emotional experience. Journal of Social and Personal Relationships, 2015. 32(6): p. 757-787.

46. Peters, K. and Y. Kashima, From social talk to social action: Shaping the social triad with emotion sharing. Journal of Personality and Social Psychology, 2007. 93(5): p. 780-797.

47. Peters, K., et al., Gossiping about deviance: Evidence that deviance spurs the gossip that builds bonds. Psychological Science, 2017: p. Advance online publication. 
48. Peters, K. and Y. Kashima, Bad habit or social good? How perceptions of gossiper morality are related to gossip content. European Journal of Social Psychology, 2015. 45(6): p. 784798.

49. Yzerbyt, V., T. Kuppens, and B. Mathieu, When talking makes you feel like a group: The emergence of group-based emotions. Cognition and Emotion, 2016. 30(1): p. 33-50.

50. Livingstone, A.G., et al., "Fury, us": Anger as a basis for new group self-categories. Cognition and Emotion, 2016. 30(1): p. 183-192. 


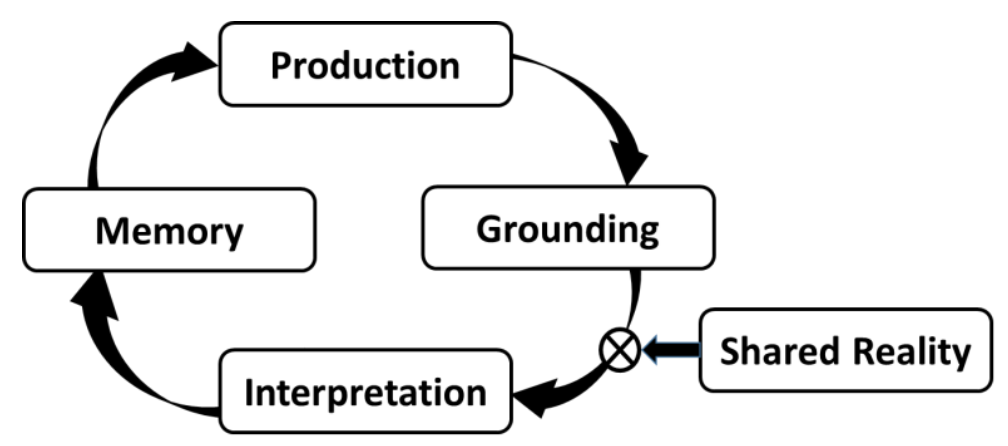

Figure 1: Cultural dynamics and shared reality. Shared reality process tags the grounded information as socially verified, and therefore the information is interpreted as a "fact" usable in the future in different contexts.

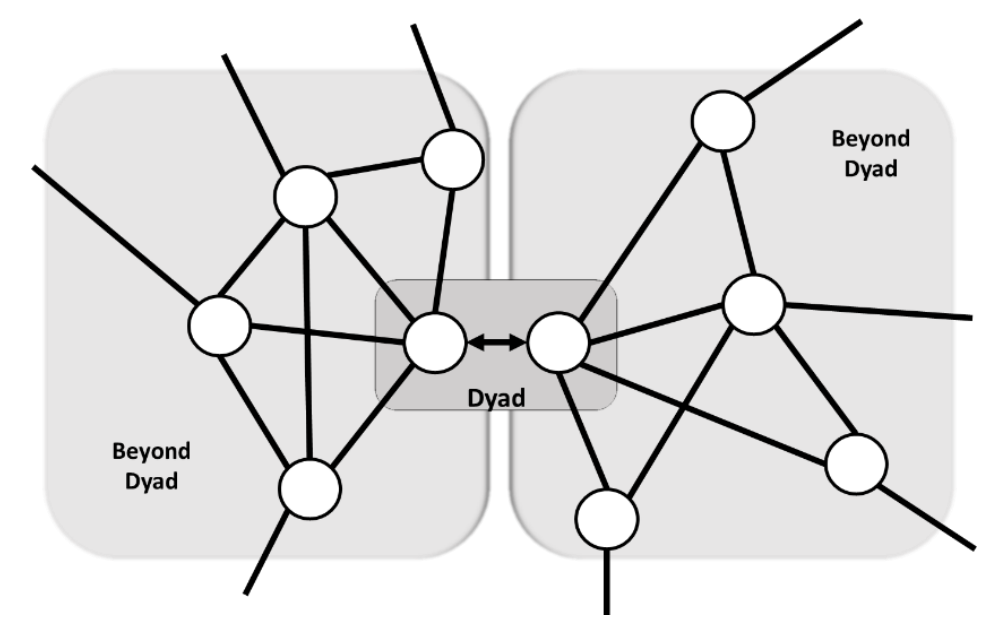

Figure 2: Cultural dynamics in social networks. Cultural information may be grounded in a dyad and its shared reality is established via mutual recognition of shared psychological responses; it then can drive further diffusion of information through social networks beyond the dyad.

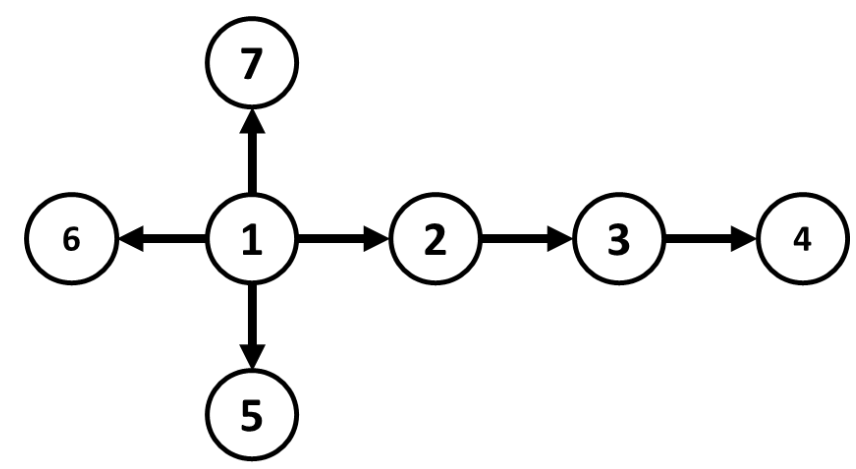

Figure 3: Experimental paradigms for cultural dynamics and social network structure. Different experimental paradigms can be used to simulate different social network structures. The method of serial reproduction simulates a chain: person 1 receives the stimulus and reproduces it to person 2 , who then in turn communicates to person 3 , etc. The method of repeated reproduction simulates a star: person 1 reproduces the stimulus to person 2 , person 5 , person 6 , and so on. 